Rev. Mat. Iberoamericana 26 (2010), no. 1, 239-259

\title{
Taylor Formula on step two Carnot Groups
}

\section{Gabriella Arena, Andrea O. Caruso and Antonio Causa}

\begin{abstract}
In the setting of step two Carnot groups we give an explicit representation of Taylor polynomial in terms of a suitable basis of the real vector space of left invariant differential operators, acting pointwisely on monomials like the ordinary Euclidean iterated derivations.
\end{abstract}

\section{Introduction}

In this article we deal with the expression of Taylor formula for real valued functions defined on a step two Carnot group $\mathbb{G}$. According to the definition, that goes back to Folland and Stein where it is given for general homogeneous Lie groups (see [6]), a polynomial $P_{m}$ of homogeneous degree $m$ at most on $\mathbb{G}$ is said to be the $m^{\text {th }}$ Taylor polynomial of a given function $f \in C_{\mathrm{H}}^{m}(\mathbb{G})$ at a fixed point $c \in \mathbb{G}$, if it realizes a contact of order $m$ with the function $f$ at $c$, that is, if $X^{I}\left(P_{m}-f\right)(c)=0$, for any iterated derivation $X^{I}$ of the canonical Poincarè-Birkhoff-Witt basis (see Definition 2), with homogeneous degree $m$ at most. The well-posedness of the definition relies on the existence of a linear isomorphism $L$ between the vector space of polynomials and the vector space of left invariant differential operators on $\mathbb{G}$ (see Remark 2). Although the definition of Taylor polynomial is quite straightforward, several difficulties of computational kind occur if one wants to write it down explicitly; indeed the polynomial valued vector fields of the Lie algebra $\mathfrak{g}$ of $\mathbb{G}$, do not commute in general, so it is not an easy task to invert the upper triangular matrix associated to $L$, for a fixed degree $m$. We attack the problem in an alternative and more theoretical way. For the sake of clarity we deal first with the simplest Carnot group $\mathbb{G}=\mathbb{H}^{1}$; in this setting, general considerations about the Taylor series expansion of a given analytic function $f$ on a 3 -dimensional analytic Lie group $\mathbb{G}$, suggest the 
expression for the $m^{\text {th }}$ Taylor polynomial of $f$ in terms of suitable symmetrized $k$-derivations (see Definition 9). Some combinatorial considerations allow us to analyze the pointwise behaviour of these symmetrized $k$-derivations when applied to monomials: we prove that their action on monomials imitates, at the central point $c$, the one of Euclidean iterated derivations (see Proposition 1). In turn, such symmetrized $k$-derivations form a basis of the vector space of differential operators on $\mathbb{G}$ and also the Taylor polynomial introduced in Definition 9, realizes a contact of order $m$ with the function $f$ at $c$ (see point ii) of Theorem 1 and Theorem 2). In support of these results, we also exhibit some algebraic considerations on the linear generators of the free Lie algebra $\mathbb{K}\langle X, Y\rangle$, investigating several sets of generators for the real vector space of left invariant differential operators on $\mathbb{G}$. In Section 4, concluding the paper, we extend all the previous considerations to the case of step two Carnot groups.

We stress that, after the paper was submitted, we knew of the investigation devoted to the Taylor polynomial in [4, Chapter 20] that, up to now and to our knowledge, seems to be the major reference text, jointly with the older [6], on calculus in general homogeneous Lie groups. Roughly speaking, the authors represent the $m^{t h}$ Taylor polynomial as the sum of all $k$-derivations of the kind $Z_{J_{k}}$ (see Definition 2) up to degree $m$ (see Corollary 20.3 .12 in [4]); nevertheless the proof that this polynomial is effectively the Taylor polynomial is done by verifying iii) of Theorem 1, and so they do not tackle the problem of determining the action of iterated vector fields on single monomials, which is exactly the core of this note. We also observe that the $2^{\text {th }}$ Taylor polynomial in the setting of the Heisenberg group $\mathbb{H}^{1}$ appeared previously in [3].

Finally, we point out that the problem of finding the explicit expression of the Taylor polynomial, raised to our attention when dealing with the generalization to the case of Carnot groups of the well known Whitney extension theorem of a given function $f$ of class $C^{m}(F)$, outside of a closed subset $F \subset \mathbb{R}^{n}$ (see $\left.[12,5,1]\right)$.

Acknowledgements. The first two authors wish to thank G. Citti, F. Serra Cassano and R. Serapioni for all the interesting discussions that introduced them into these arguments. In particular they are deeply grateful to the last one for the time he spent generously in fruitful discussions. Moreover, the second author wishes to thank the Department of Mathematics of Trento for the kind hospitality during the preparation of the paper.

The results in this paper have been announced at the the workshop Equazioni a derivate parziali: aspetti metodologici, modellistica, applicazioni held on July 2005 in Ragusa Ibla (Ragusa, Italy) and at the Meeting on Geometric Measure Theory and Calculus of Variations held on February 2006 in Levico Terme (Trento, Italy). 


\section{Notation and Preliminaries}

\subsection{The stratified structure of step two Carnot groups}

A step two Carnot group is a connected and simply connected nilpotent Lie group of stratified type, i.e., its Lie algebra denoted by $\mathfrak{g}$, admits a decomposition as direct sum of two vector subspaces. Namely, $\mathfrak{g}=V_{1} \oplus V_{2}$ where $V_{2}=\left[V_{1}, V_{1}\right], V_{1}$ is called the horizontal slice and its vector fields the horizontal vector fields on $\mathbb{G}$. Evidently, such an algebra is nilpotent of step two by definition or, equivalently, $V_{2}$ is included in the center of $\mathfrak{g}$. The stratified structure of $\mathfrak{g}$ gives rise to a family of mappings $\left\{\gamma_{\lambda}\right\}_{\lambda \in \mathbb{R}}$ of $\mathfrak{g}$ called dilations, which turn to be a group of homomorphims for $\lambda>0$, defined on the generators by imposing, for any $\lambda \geq 0, \gamma_{\lambda}(X)=\lambda X$ whenever $X \in V_{1}$, $\gamma_{\lambda}(T)=\lambda^{2} T$ whenever $T \in V_{2}$ and, finally, $\gamma_{-1}(Y)=-Y$ for any $Y \in \mathfrak{g}$. The family $\left\{\gamma_{\lambda}\right\}_{\lambda \in \mathbb{R}}$ can be pushed forward on $\mathbb{G}$ via the exponential map, obtaining a family of dilations on $\mathbb{G}$ by the position $\delta_{\lambda}:=\exp \circ \gamma_{\lambda} \circ \exp ^{-1}$. After, setting $l=\operatorname{dim}\left(V_{1}\right)$ and $p=\operatorname{dim}\left(V_{2}\right)$, we choose a basis of $\mathfrak{g}$ adapted to the stratification by selecting a basis of left invariant vector fields $X_{1}, \ldots, X_{l}$ of $V_{1}$ and $T_{1}, \ldots, T_{p}$ of $V_{2}$ such that $X_{i}(e)=e_{i}$ for any $i=1, \ldots, l$, and $T_{j}(e)=e_{l+j}$ for any $j=1, \ldots, p$, where $e$ is the identity of $\mathbb{G}$ and $\left\{e_{h}\right\}_{h=1, \ldots, n}$ denotes the standard basis of $\mathbb{R}^{n}, n=l+p$. Relatively to this basis we write $z=(x, t) \equiv\left(x_{1}, \ldots, x_{l}, t_{1}, \ldots, t_{p}\right)$, or also $z \equiv\left(z_{h}\right), h=1, \ldots, n$, where such coordinates of $z \in \mathbb{G}$ comes from the exponential first kind representation $z=\exp \left(\sum_{i=1}^{l} x_{i} X_{i}+\sum_{j=1}^{p} t_{j} T_{j}\right)$; in particular, for $\lambda \geq 0, \delta_{\lambda}(z)=\left(\lambda x, \lambda^{2} t\right)$. With respect to such a given system of coordinates, it is not difficult to verify (see for instance Theorem 3.2.2 in [4]) that, setting $z=(x, t), w=(\xi, \tau)$ with $x, \xi \in \mathbb{R}^{l}$ and $t, \tau \in \mathbb{R}^{p}$, then

$$
z w=\left(x+\xi, t+\tau+\frac{1}{2}\langle B x, \xi\rangle\right)
$$

where, $\langle B x, \xi\rangle$ denotes the $p$-tuple $\left(\left\langle B^{(1)} x, \xi\right\rangle, \ldots,\left\langle B^{(p)} x, \xi\right\rangle\right)$ for suitable $l \times l$ independent skew-symmetric matrices $B^{(1)}, \ldots, B^{(p)}$. It follows easily that the identity $e \in \mathbb{G}$ is identified with $0 \in \mathbb{R}^{n}$ and that the inverse of an element $z=(x, t)$ is identified with $-z=(-x,-t)$.

\subsection{Homogeneity degree and $k$-derivations}

Definition 1 We say that a function $f: \mathbb{G} \rightarrow \mathbb{R}$ is homogeneous of degree $\alpha \in \mathbb{R}$ if, $f\left(\delta_{\lambda}(x)\right)=\lambda^{\alpha} f(x)$ for any $\lambda>0$. We say that a left invariant differential operator $D$ on $\mathbb{G}$ is homogeneous of degree $\alpha \in \mathbb{R}$ if, $D(f \circ$ $\left.\delta_{\lambda}\right)(x)=\lambda^{\alpha}\left(D f \circ \delta_{\lambda}\right)(x)$, for any smooth function $f$ and for any $\lambda>0$. 
Clearly, if $D$ is a left invariant differential operator homogenous of degree $\alpha$ and $f$ is a function homogeneous of degree $\beta$, then $D f$, if defined, is a function homogeneous of degree $\beta-\alpha$ and $f D$ is a left invariant differential operator homogenous of degree $\alpha-\beta$; moreover if $D_{1}, D_{2}$ are left invariant differential operators, homogenous of degree $\alpha$ and $\beta$ respectively, then $D_{1} D_{2}$ is a left invariant differential operator homogenous of degree $\alpha+\beta$. Arguing as in Proposition 2.2. of [8] it is possible to verify that

$$
\begin{aligned}
& X_{i}=\partial_{x_{i}}+\frac{1}{2}\left\langle(B x)_{i}, \nabla_{t}\right\rangle \text { for each } i=1, \ldots, l \\
& T_{j}=\partial_{t_{j}} \text { for each } j=1, \ldots, p
\end{aligned}
$$

where $\nabla_{t}:=\left(\partial_{t_{1}}, \ldots, \partial_{t_{p}}\right),(B x)_{i}:=\left(\left(B^{(1)} x\right)_{i}, \ldots,\left(B^{(p)} x\right)_{i}\right)$ and $\left(B^{(k)} x\right)_{i}$ is the $i^{\text {th }}$ component of $B^{(k)} x$. Consequently, each $X_{i}$ is homogeneous of degree one and each $T_{j}$ is homogeneous of degree two.

Definition 2 (Derivations and multi-indexes) A left invariant differential operator of the kind $Z_{J_{k}}=X_{i_{1}} \cdots X_{i_{m}} T_{j_{1}} \cdots T_{j_{r}}$, where $k$ is a nonnegative integer, $m+r=k$ and $1 \leq i_{1}, \ldots, i_{m} \leq l, 1 \leq j_{1}, \ldots, j_{r} \leq p$, is called $k$-derivation; in this case, setting $J_{k}=\left(i_{1}, \ldots, i_{m}, j_{1}, \ldots, j_{r}\right)$, the numbers $\left|J_{k}\right|=m+r$ and $d\left(J_{k}\right)=m+2 r$ are called respectively the order and the degree of $X_{J_{k}}$ and $J_{k}$. In particular, a left invariant differential operator of the kind $Z_{H_{k}}=X_{i_{1}} \cdots X_{i_{k}}$, where $1 \leq i_{1}, \ldots, i_{k} \leq l$, is called horizontal $k$-derivation and in this case we have $\left|H_{k}\right|=d\left(H_{k}\right)=k$.

$A$ left invariant differential operator of the kind $Z^{I}=X_{1}^{i_{1}} \cdots X_{l}^{i_{l}} T_{1}^{j_{1}} \cdots T_{p}^{j_{p}}$ where, for $n=l+p, I=\left(i_{1}, \ldots, i_{l}, j_{1}, \ldots, j_{p}\right)$ is a $n$-tuple of non negative integers, will be called a derivation of the canonical basis of BPW (short for Birkhoff-Poincarè-Witt, see next Remark 1) -briefly canonical derivationof order $|I|=\sum_{h=1}^{l} i_{h}+\sum_{h=1}^{p} j_{h}$ and degree $d(I)=\sum_{h=1}^{l} i_{h}+\sum_{h=1}^{p} 2 j_{h}$; as before, the numbers $|I|, d(I)$ are also called the order and the degree of the multi-index $I$.

In both the cases, a 0-derivation, denoted equivalently with the symbols $Z_{J_{0}}, Z_{H_{0}}$ and $Z^{I}$, where $I$ is the null $n$-tuple, is just the identity operator.

Finally, we shall denote by $\left\{Z_{\alpha}^{i}\right\}_{\substack{i \in A_{i} \\ \text { a }}}$ a given basis for the real vector space of all left invariant differential operators on $\mathbb{G}$; so, for any $i \in \mathbb{N}$, $A_{i}$ is a set of indexes for the basis $\left\{Z_{\alpha}^{i}\right\}_{\alpha \in A_{i}}$ of the finite dimensional real vector space of left invariant differential operators of homogeneous degree $i$ (in particular $\left\{Z_{\alpha}^{1}\right\}_{\alpha \in A_{1}}$ is chosen adapted to the stratification of $\mathfrak{g}$ ).

Horizontal $k$-derivations are also called nonholonomic partial derivatives of order $k$, see for instance [2]. 
Remark 1 (Birkhoff-Poincaré-Witt Theorem) Recall that the Birkhoff-Poincaré-Witt theorem ensures that the set of all canonical derivations $Z^{I}$ constitutes a basis for the algebra of all left invariant differential operators on $\mathbb{G}$ (see, for instance, [11]). In particular, taking into account the stratified structure of $\mathfrak{g}$, the following spanning relationships easily hold

$$
\begin{aligned}
\operatorname{Span}_{\mathbb{R}}\left(\left\{Z_{I_{k}}\right\}\right)_{d\left(I_{k}\right)=k} & =\operatorname{Span}_{\mathbb{R}}\left(\left\{Z_{H_{k}}\right\}\right)=\operatorname{Span}_{\mathbb{R}}\left(\left\{Z^{I}\right\}\right)_{d(I)=k} \\
& =\operatorname{Span}_{\mathbb{R}}\left(\left\{Z_{\alpha}^{k}\right\}\right)_{\alpha \in A_{k}} .
\end{aligned}
$$

\subsection{Polynomials on Carnot groups}

Definition 3 A function $P: \mathbb{G} \rightarrow \mathbb{R}$ is called a polynomial on $\mathbb{G}$ if $P \circ \exp ^{-1}$ is a polynomial on the vector space $\mathfrak{g}$.

More precisely, according to the previous notation $z=\left(z_{h}\right) \in \mathbb{G}$, if $\left(\zeta_{h}\right):=$ $\left(\omega_{i}, \eta_{j}\right) \in \mathfrak{g}^{*}$ denotes the dual basis of $\left(Z_{h}\right):=\left(X_{i}, T_{j}\right)$, then, for any $h=1, \ldots, n$, we have $\zeta_{h} \circ \exp ^{-1}(z)=z_{h}$. So, if $I$ is a multi-index as in Definition 2, the general monomial can be written as $\mathrm{m}^{I}(z)=z^{I}=$ $x_{1}^{i_{1}} \cdots x_{l}^{i_{l}} t_{1}^{j_{1}} \cdots t_{p}^{j_{p}}$. Note that $\mathrm{m}^{I}\left(\delta_{\lambda}(x)\right)=\lambda^{d(I)} \mathrm{m}^{I}(x)$, so the monomial $\mathrm{m}^{I}$ is of homogeneous degree $d(I)$. Consequently, a basis for the real vector space of polynomials of degree $m$ at most is the set of all monomials of the kind $\mathrm{m}^{I}$ for all $n$-tuples $I$ such that $d(I) \leq m$, hence a general polynomial $P$ of homogeneous degree $m$ is $P=\sum_{d(I) \leq m} a_{I} \mathrm{~m}^{I}$, for some $a_{I} \in \mathbb{R}$ such that $\max \left\{d(I): a_{I} \neq 0\right\}=m$. In what follows we shall denote by $P_{m}$ a polynomial $P$ for which $\max \left\{d(I): a_{I} \neq 0\right\} \leq m$. We stress that, as in the Euclidean case, the homogeneous degree of a given polynomial depends neither on the fixed basis adapted to the stratification nor on the translation in the group (see for instance Proposition 1.25 in [6]).

\subsection{Carnot-Caratheodory metrics}

In every Carnot group a natural sub-Riemannian distance between two given points $x, y \in \mathbb{G}$ can be introduced as the infimum of all times $s$ for which there exists an absolutely continuous horizontal curve joining the points, i.e., a curve $\gamma:[0, s] \rightarrow \mathbb{G}$ such that $\dot{\gamma}=\sum_{j=1}^{l} \mu_{j} X_{j}(\gamma)$ a.e., for some measurable vector function $\mu:[0, s] \rightarrow \mathbb{R}^{l},\|\mu\|_{\infty} \leq 1, \gamma(0)=x, \gamma(s)=y$. Such horizontal curves do exist by the Hörmander bracket generating condition, so the distance $d$ is finite and such that, for any fixed Euclidean compact set $K \subset \mathbb{G}$, there exists a constant $C=C(K)$ for which $\frac{1}{C}\|x-y\| \leq d(x, y) \leq$ $C\|x-y\|^{\frac{1}{k}}$, for any $x, y \in K$, where $\|\cdot\|$ denotes the Euclidean norm. This fact proves that the Euclidean topology coincides with the one induced by $d$ but, in general, the two distances are not equivalent. Nevertheless, $d$ shares with 
the Euclidean metric the (left) translation invariance and the homogeneity of degree one with respect to the dilations $\delta_{\lambda}$, i.e., $d(z x, z y)=d(x, y)$ and $d\left(\delta_{\lambda}(x), \delta_{\lambda}(y)\right)=|\lambda| d(x, y)$, for any $x, y, z \in \mathbb{G}$ and for any $\lambda \in \mathbb{R}$.

On any Carnot group $\mathbb{G}$ there exist several quasi-metrics equivalent to the Carnot-Caratheodory one, but much more easier to handle (refer to [6] and [8] for more details). In what follows, we do not need a particular quasimetric or metric, so we will write $d$ to denote any one of the distance function described above satisfying the translation invariance and the homogeneity of degree one.

\subsection{Calculus on Carnot groups and Taylor polynomials}

The notion of Lie derivative meets sub-Riemannian geometry in the following definition.

Definition 4 Let $f: \mathbb{G} \rightarrow \mathbb{R}, c \in \mathbb{G}, X \in V_{1}$ and let $v=\exp (X)$. We say that the function $f$ is differentiable along $X$ at the point $c$ if the function $\mathbb{R} \ni \lambda \rightarrow f\left(c \delta_{\lambda}(v)\right) \in \mathbb{R}$ is differentiable at the point $\lambda=0$; in this case we write $X f(c)$ for such a limit.

Following Folland and Stein (see [6]), we recall the definition of function of class $C_{\mathrm{H}}^{m}(\mathbb{G})$ on Carnot groups.

Definition 5 Let $f: \mathbb{G} \rightarrow \mathbb{R}$. We say that $f \in C_{\mathrm{H}}^{1}(\mathbb{G})$ if $X_{i} f$ exist and are continuous at each point of $\mathbb{G}$, for every $i=1, \ldots, l$. Moreover, for any non-negative integer $m$, we say that $f \in C_{\mathrm{H}}^{m}(\mathbb{G})$, if $X_{H_{k}} f$ exist and are continuous at each point of $\mathbb{G}$, for every horizontal $k$-derivation $X_{H_{k}}$ such that $0 \leq k \leq m$.

Clearly, according to Remark 1 , the definition of function of class $C_{\mathrm{H}}^{m}(\mathbb{G})$ can be equivalently done with anyone of the $k$-derivations given in Definition 2 . Moreover, it can be verified that $f \in C_{\mathrm{H}}^{m}(\mathbb{G})$ if and only if anyone of the $(k$ or $|I|)$-derivations homogeneous of degree $m$ at most exist in distributional sense and are continuous at each point of $\mathbb{G}$.

Finally, we recall the definition of Taylor polynomial of a function $f \in$ $C_{\mathrm{H}}^{m}(\mathbb{G})$, according to $[6]$.

Definition 6 Let $m$ be a non-negative integer, $c \in \mathbb{G}$ and $f \in C_{\mathrm{H}}^{m}(\mathbb{G})$. A polynomial $P_{m}$ is called the (left) Taylor polynomial of $f$ at the point $c$, if $X^{I}(P-f)(c)=0$, for any $|I|$-derivation of the BPW basis such that $0 \leq d(I) \leq m$.

Remark 2 Taking into account that $X^{I}$ is a left invariant differential operator, the well posedness of this definition relies on Proposition 1.30 of [6]. 
More precisely, denoting with $\mathcal{P}_{m}$ and $\mathcal{D}_{m}$ respectively the vector spaces of all polynomials and of all left invariant differential operators on $\mathbb{G}$, both of homogeneous degree $d(I) \leq m$, the linear mapping $L: \mathcal{P}_{m} \rightarrow \mathcal{D}_{m}$ defined through the position $L\left(P_{m}\right)=\sum_{d(I)=0}^{m}\left(X^{I} P(0)\right) X^{I}, \forall P_{m} \in \mathcal{P}_{m}$, comes to be an isomorphism; in particular the matrix $M$ associated to $L$, with respect to the two BPW bases $\left\{x^{I}\right\}_{d(I)=0,1, \ldots, m}$ and $\left\{X^{I}\right\}_{d(I)=0,1, \ldots, m}$ is upper triangular with non zero diagonal. In the simplest Heisenberg group $\mathbb{H}^{1}$ (see examples in Subsection 2.5), we have $L(x)=X, L(y)=Y$ but $L(t)=T-2 X Y$.

So, if we want to write down the Taylor polynomial of a given function $f \in C_{\mathrm{H}}^{m}(\mathbb{G})$, we must determine the action of $L^{-1}$, i.e., write explicitly the matrix $M^{-1}$ : unfortunately this is not an easy task in the general case. In the next sections we will tackle this difficulty exhibiting, in the case of step two Carnot groups, an alternative basis for $\mathcal{D}_{m}$ which will fill this gap.

For the sake of completeness, and according to notations introduced in Subsection 2.2, we state the following well known facts in a form useful to our purposes, sketching some of the proofs.

Theorem 1 Let $m$ be a non negative integer, $c \in \mathbb{G}, f \in C_{\mathrm{H}}^{m}(\mathbb{G})$ and $P_{m}$ a given polynomial of homogeneous degree $m$ at most. Then, according to notation introduced in Definition 2, the following facts are equivalent:

i) $P_{m}$ is the $m^{\text {th }}$ Taylor polynomial of $f$ at $c$;

ii) $Z_{\alpha}^{i}\left(P_{m}-f\right)(c)=0$, for any $i=0, \ldots, m$ and for any $\alpha \in A_{i}$;

iii) $\left(P_{m}-f\right)(x)=o[d(x, c)]^{m}$ as $x \rightarrow c$.

Proof. i) $\Longleftrightarrow$ ii) follows immediately from Remark 1. i) $\Longrightarrow$ iii) follows immediately from the stratified Taylor inequality proved in Theorem 1.42 of [6]. iii) $\Longrightarrow i$ ): It suffices to verify that if $Q_{m}$ is a polynomial of homogeneous degree $m$ at most such that $Q_{m}(x)=o[d(x, 0)]^{m}$ as $x \rightarrow 0$, then $Q_{m}$ is identically zero. Indeed, let $Q_{m}=\sum_{d(I) \leq m} a_{I} \mathrm{~m}^{I}$ and let us verify, by induction, that $a_{I}=0$ for all $I$ such that $d(I) \leq m$. This is trivial when $d(I)=0$; assuming that $a_{I}=0$ for all $I$ such that $d(I)=k<m$, we can write $Q_{m}=\sum_{d(I)=k+1} a_{I} \mathrm{~m}^{I}+\sum_{k+1<d(I) \leq m} a_{I} \mathrm{~m}^{I}$. Fix $p \in \mathbb{G}, p \neq 0$. Then $\delta_{\lambda}(p) \rightarrow 0$ if and only if $\lambda \rightarrow 0$ and, recalling that $d$ is homogeneous of degree one, we have

$$
\frac{\sum_{d(I)=k+1} a_{I} \mathrm{~m}^{I}(p)}{[d(p, 0)]^{k+1}}=\frac{Q_{m}\left(\delta_{\lambda}(p)\right)}{\left[d\left(\delta_{\lambda}(p), 0\right)\right]^{k+1}}-\frac{\sum_{k+1<d(I) \leq m} \lambda^{d(I)} a_{I} \mathrm{~m}^{I}(p)}{\lambda^{k+1}[d(p, 0)]^{k+1}} \rightarrow 0 .
$$

Thanks to the arbitrariness of $p$ it follows that $a^{I}=0$ for any $I$ such that $d(I)=k+1$, as desired. To conclude the proof observe that if $P_{m}^{\prime}$ is 
the Taylor polynomial of $f$ at the point $c$, then, denoting by $\tau_{c}$ the left translation pointed at $c$ we have $\left(P_{m}-P_{m}^{\prime}\right) \circ \tau_{c}^{-1}(x)=o[d(x, 0)]^{m}$ as $x \rightarrow 0$, which implies $P_{m}=P_{m}^{\prime}$.

We conclude this subsection observing that, thanks to the left invariance of the vector fields, for any $f \in C_{\mathrm{H}}^{m}(\mathbb{G})$, for any $c \in \mathbb{G}$ and for any $|I|$-derivation of the canonical basis of BPW, we have $X^{I} f(c)=X^{I}\left(f \circ \tau_{c}\right)(0)$ so, in the next sections, we will look for the Taylor polynomial at the identity of $\mathbb{G}$.

\section{Examples}

The paradigmatic example of step two groups is the Heisenberg group $\mathbb{H}^{n}$ associated to the Lie algebra $\mathfrak{h}^{n}=\mathfrak{h} \oplus \mathfrak{v}$ whose linearly independent generators $X_{1}, \ldots, X_{n}, Y_{1}, \ldots, Y_{n}, T$ satisfy the conditions $\mathfrak{h}=\operatorname{Span}_{\mathbb{R}}\left(\left\{X_{j}, Y_{j}\right\}_{j=1, \ldots, n}\right)$, $\mathfrak{v}=\operatorname{Span}_{\mathbb{R}}\{T\}$ and the only non zero brackets are $\left[X_{j}, Y_{j}\right]=a T$, for any $j=$ $1, \ldots, n$ and for some fixed real number $a \neq 0$. If $p=\left(x_{1}, \ldots, x_{n}, y_{1}, \ldots, y_{n}, t\right)$, $q=\left(x_{1}^{\prime}, \ldots, x_{n}^{\prime}, y_{1}^{\prime}, \ldots, y_{n}^{\prime}, t^{\prime}\right) \in \mathbb{H}^{n}$, than the group product reads $p q=$ $\left(x_{1}+x_{1}^{\prime}, \ldots, x_{n}+x_{n}^{\prime}, y_{1}+y_{1}^{\prime}, \ldots, y_{n}+y_{n}^{\prime}, t+t^{\prime}+\frac{a}{2} \sum_{j=1}^{n}\left(x_{j} y_{j}^{\prime}-x_{j}^{\prime} y_{j}\right)\right)$; it follows that $X_{j}=\frac{\partial}{\partial x_{j}}-\frac{a}{2} y_{j} \frac{\partial}{\partial t}, Y_{j}=\frac{\partial}{\partial y_{j}}+\frac{a}{2} x_{j} \frac{\partial}{\partial t}$ and $T=\frac{\partial}{\partial t}$. In this paper we follow notation as in [7], choosing $a=-4$.

The Heisenberg group $\mathbb{H}^{n}$ is actually the prototype of a general class of step two Carnot groups, the so called H-type groups (see [9]). More precisely, let $\mathfrak{g}$ be a Lie algebra nilpotent of step two endowed with an inner product $\langle\cdot, \cdot\rangle$. Let $\mathfrak{z}$ be the center of $\mathfrak{g}$. For any $v \in \mathfrak{z}^{\perp}, \operatorname{ad}_{v}$ is a $\mathfrak{z}^{- \text {valued }}$ linear mapping: set $\mathfrak{k}_{v}=\operatorname{ker}\left(\operatorname{ad}_{v}\right) \cap \mathfrak{z}^{\perp}$ so that $\mathfrak{z}^{\perp}=\mathfrak{k}_{v} \oplus \mathfrak{k}_{v}^{\perp}$. Then $\mathfrak{g}$ is said to be of $\mathrm{H}$-type if $\operatorname{ad}_{v}: \mathfrak{k}_{v}^{\perp} \rightarrow \mathfrak{z}$ is a surjective isometry for every vector $v$ such that $\langle v, v\rangle=1$ and, in this case, the corresponding group is said to be a group of type $\mathrm{H}$. It can be verified that these conditions are equivalent to say that, according to the general form of the group law, each $B^{(i)}$ is also an orthogonal matrix and, for any $i_{1}$, $i_{2}$, we have $B^{\left(i_{1}\right)} B^{\left(i_{2}\right)}+B^{\left(i_{2}\right)} B^{\left(i_{1}\right)}=0$.

\section{Taylor Formula on $\mathbb{H}^{1}$}

In this section we propose an explicit expression for the Taylor polynomial $P_{m, f}$ : we restrict the argument to the case $\mathbb{H}^{1}$; indeed it will be clear at the end of this subsection how to extend the formula and relative proofs to the case $\mathbb{H}^{n}$. We are going to prove that (see the following Theorem 2) $P_{m, f}$ is the unique polynomial on $\mathbb{H}^{1}$ for which the equivalent facts of Theorem 1 hold. In order to find $P_{m, f}$, we begin by recovering Taylor formula using standard arguments of calculus in Lie groups. We assume first that $\mathbb{G}$ is an analytic Lie group and that $f$ is defined and analytic around the identity $e \in \mathbb{G}$ that we identify with $0 \in \mathbb{R}^{n}$. For $X, Y, T \in \mathfrak{g}$, the following 
Taylor series holds (see for instance [11, Theorem 2.12.3], see also the Appendix in [10]):

$$
\begin{aligned}
f(x, y, t) & =\sum_{k=0}^{\infty} \frac{1}{k !}\left[(x X+y Y+t T)^{k} f\right](0)= \\
& =\sum_{k=0}^{\infty}\left[\sum_{\substack{n_{1}, n_{2}, n_{3}=0,1, \ldots \\
n_{1}+n_{2}+n_{3}=k}} \frac{\left(\frac{\sigma\left(X^{n_{1}}, Y^{n_{2}}, T^{n_{3}}\right)}{\left(n_{1}+n_{2}+n_{3}\right) !} f\right)(0)}{n_{1} ! n_{2} ! n_{3} !} x^{n_{1}} y^{n_{2}} t^{n_{3}}\right],
\end{aligned}
$$

where the meaning of the symbol $\sigma\left(X^{n_{1}}, Y^{n_{2}}, T^{n_{3}}\right)$ relies on the following definition.

Definition 7 Let $a_{1}, \ldots, a_{r}$ be given elements of a (not necessarily commutative) ring $A$. Set

$$
\sigma\left(a_{1}, \ldots, a_{r}\right)=\sum_{\pi \in S_{r}} a_{\pi(1)} \cdots a_{\pi(r)}
$$

where $\pi \in S_{r}$ denotes an element of the symmetric group over $\{1, \ldots, r\}$.

With the aid of Definition 7 , for any fixed $n_{1}, n_{2}, n_{3}=0,1, \ldots$, setting $X_{1}=$ $\cdots=X_{n_{1}}=X, X_{n_{1}+1}=\cdots=X_{n_{1}+n_{2}}=Y, X_{n_{1}+n_{2}+1}=\cdots=X_{n_{1}+n_{2}+n_{3}}=T$, we can define

$$
\sigma\left(X^{n_{1}}, Y^{n_{2}}, T^{n_{3}}\right)=\sigma\left(X_{1}, \ldots, X_{n_{1}+n_{2}+n_{3}}\right) .
$$

Now having in mind the case $\mathbb{G}=\mathbb{H}^{1}$ one observes that, relatively to $\left(T S_{f}\right)$, the degree of the monomial $x^{n_{1}} y^{n_{2}} t^{n_{3}}$ appearing in the $k^{t h}$-sum is $n_{1}+n_{2}+$ $2 n_{3}$ which, in general, is different from $k$; so it appears quite natural to rewrite $\left(T S_{f}\right)$ as follows

$$
f(x, y, t)=\sum_{k=0}^{\infty}\left[\sum_{\substack{n_{1}, n_{2}, n_{3}=0,1, \ldots \\ n_{1}+n_{2}+2 n_{3}=k}} \frac{\left(\frac{\sigma\left(X^{n_{1}}, Y^{n_{2}}, T^{n_{3}}\right)}{\left(n_{1}+n_{2}+n_{3}\right) !} f\right)(0)}{n_{1} ! n_{2} ! n_{3} !} x^{n_{1}} y^{n_{2}} t^{n_{3}}\right] .
$$

All these considerations suggest the right candidate for the Taylor polynomial of a given $f \in C_{\mathrm{H}}^{m}\left(\mathbb{H}^{1}\right)$ :

Definition 8 For any $f \in C_{\mathrm{H}}^{m}\left(\mathbb{H}^{1}\right)$, we set

$$
\left(P_{m, f}\right) \quad P_{m, f}(x, y, t)=\sum_{k=0}^{m}\left[\sum_{\substack{n_{1}, n_{2}, n_{3}=0,1, \ldots \\ n_{1}+n_{2}+2 n_{3}=k}} \frac{\left(\frac{\sigma\left(X^{n_{1}}, Y^{n_{2}}, T^{n_{3}}\right)}{\left(n_{1}+n_{2}+n_{3}\right) !} f\right)(0)}{n_{1} ! n_{2} ! n_{3} !} x^{n_{1}} y^{n_{2}} t^{n_{3}}\right] .
$$


Remark 3 Let $\operatorname{Sym}\left(X^{n_{1}}, Y^{n_{2}}, T^{n_{3}}\right)$ be the sum of all $k$-iterated vector fields, each of them containing $n_{1}$ times the derivation $X, n_{2}$ times the derivation $Y$, and $n_{3}$ times the derivation $T$, then it is clear that $\operatorname{Sym}\left(X^{n_{1}}, Y^{n_{2}}, T^{n_{3}}\right)$ $=\frac{\sigma\left(X^{n_{1}}, Y^{n_{2}}, T^{n_{3}}\right)}{n_{1} ! n_{2} ! n_{3} !}$. Another useful property of this symmetrizing operator is the following. For any $n_{1}, n_{2}=1,2, \ldots$, we have

$$
\begin{aligned}
\operatorname{Sym}\left(X^{n_{1}}, Y^{n_{2}}\right) & =\operatorname{Sym}\left(X^{n_{1}-1}, Y^{n_{2}}\right) X+\operatorname{Sym}\left(X^{n_{1}}, Y^{n_{2}-1}\right) Y \\
& =X \operatorname{Sym}\left(X^{n_{1}-1}, Y^{n_{2}}\right)+Y \operatorname{Sym}\left(X^{n_{1}}, Y^{n_{2}-1}\right) .
\end{aligned}
$$

Both these equalities follow immediately from the consideration that the differential operators appearing in the second (resp. the third) member are all different by construction and, moreover, by observing that, by the general binomial identity $\frac{\left(n_{1}+n_{2}\right) !}{n_{1} ! n_{2} !}=\left(\begin{array}{c}n_{1}+n_{2}-1 \\ n_{1}\end{array}\right)+\left(\begin{array}{c}n_{1}+n_{2}-1 \\ n_{2}\end{array}\right), n_{1}, n_{2}=1,2, \ldots$, both the first and the second (resp. the third) members, have the same number of elements.

Now we want to rewrite $\left(P_{m, f}\right)$ as a sum of formal powers i.e. as in the first series of $\left(T S_{f}\right)$. We observe that in $(x X+y Y+t T)^{k}$ it is natural to see the summand containing $n_{1}$ times the derivation $X, n_{2}$ times the derivation $Y$ and $n_{3}$ times the derivation $T$, where $n_{1}+n_{2}+2 n_{3}=k$, as a unique $\left(n_{1}+n_{2}+n_{3}\right)^{t h}$-iterated derivation, more precisely as $\frac{n_{1} ! n_{2} ! n_{3} !}{\left(n_{1}+n_{2}+n_{3}\right) !}$. $\operatorname{Sym}\left(X^{n_{1}}, Y^{n_{2}}, T^{n_{3}}\right)$; so, the following definition appears quite natural.

Definition 9 Set, for any non negative integers $k, n_{1}, n_{2}, n_{3}$ such that $n_{1}+$ $n_{2}+2 n_{3}=k$,

$$
\frac{\partial^{k}}{\partial X^{n_{1}} \partial Y^{n_{2}} \partial T^{n_{3}}}=\frac{n_{1} ! n_{2} ! n_{3} !}{\left(n_{1}+n_{2}+n_{3}\right) !} \cdot \operatorname{Sym}\left(X^{n_{1}}, Y^{n_{2}}, T^{n_{3}}\right) .
$$

We call (3.1) a symmetrized $\left(n_{1}+n_{2}+n_{3}\right)$-derivation of order $k$ containing $n_{1}$-times $X, n_{2}$-times $Y$ and $n_{3}$-times $T$ (symmetrized derivation for short, when we need to specify neither the order $k$ nor the single vector fields).

Remark 4 Observe that in Definition 9 the number $k$ is the homogeneity degree of the left invariant differential operator. For instance $\partial^{0}=$ id, $\frac{\partial^{1}}{\partial X^{1}}=X, \frac{\partial^{1}}{\partial Y^{1}}=Y, \frac{\partial^{2}}{\partial T^{1}}=T$. Moreover we observe that, as in Remark 3, we have the following property for these symmetrized derivations. For any $n_{1}, n_{2}=1,2, \ldots$, we have

$$
\begin{aligned}
\frac{\partial^{n_{1}+n_{2}}}{\partial X^{n_{1}} \partial Y^{n_{2}}} & =\frac{n_{1}}{n_{1}+n_{2}} \cdot \frac{\partial^{n_{1}+n_{2}-1}}{\partial X^{n_{1}-1} \partial Y^{n_{2}}} X+\frac{n_{2}}{n_{1}+n_{2}} \cdot \frac{\partial^{n_{1}+n_{2}-1}}{\partial X^{n_{1}} \partial Y^{n_{2}-1}} Y \\
& =\frac{n_{1}}{n_{1}+n_{2}} \cdot X \frac{\partial^{n_{1}+n_{2}-1}}{\partial X^{n_{1}-1} \partial Y^{n_{2}}}+\frac{n_{2}}{n_{1}+n_{2}} \cdot Y \frac{\partial^{n_{1}+n_{2}-1}}{\partial X^{n_{1}} \partial Y^{n_{2}-1}} .
\end{aligned}
$$

Both these equalities follow immediately from ( $\left.\mathrm{S}^{\prime}\right)$. 
Now, according to Definition 9 , we can come back to formula $\left(P_{m, f}\right)$, and rewrite it as follows

$$
\begin{aligned}
& \left(P_{m, f} \text { - Second Form }\right) \\
& \quad P_{m, f}(x, y, t)=\sum_{k=0}^{m}\left[\sum_{\substack{n_{1}, n_{2}, n_{3}=0,1, \ldots \\
n_{1}+n_{2}+n_{3}=k}} \frac{\left(\frac{\partial^{k}}{\partial X^{n_{1}} \partial Y^{n_{2}} \partial T^{n_{3}}} f\right)(0)}{n_{1} ! n_{2} ! n_{3} !} x^{n_{1}} y^{n_{2}} t^{n_{3}}\right] .
\end{aligned}
$$

Remark 5 Observe that, since $T$ belongs to the center of $\mathfrak{h}^{1}$, the differential operator $\operatorname{Sym}\left(X^{n_{1}}, Y^{n_{2}}, T^{n_{3}}\right)$ satisfies the relation $\operatorname{Sym}\left(X^{n_{1}}, Y^{n_{2}}, T^{n_{3}}\right)=$ $\left(\begin{array}{c}n_{1}+n_{2}+n_{3} \\ n_{3}\end{array}\right) \cdot \operatorname{Sym}\left(X^{n_{1}}, Y^{n_{2}}\right) T^{n_{3}}$ or, equivalently, $\frac{\partial^{k}}{\partial X^{n_{1}} \partial Y^{n_{2}} \partial T^{n_{3}}}=\frac{\partial^{n_{1}+n_{2}}}{\partial X^{n_{1}} \partial Y^{n_{2}}} T^{n_{3}}$.

Remark 6 If we want to rewrite the polynomial $P_{m, f}$ as a sum of formal powers of degree $k$, we have to involve the ratio $\frac{k !}{n_{1} ! n_{2} ! n_{3} !}$, where $n_{1}+n_{2}+$ $2 n_{3}=k$. In the Euclidean case, where $k=n_{1}+n_{2}+n_{3}$, it is a polynomial coefficient of degree $k$ and it represents the number of the permutations of the objects $x, y$ and $t$ repeated respectively $n_{1}, n_{2}$ and $n_{3}$ times. Analogously, we can introduce a polynomial Newton-Leibniz formula adapted to graded coordinates in $\mathbb{H}^{1}$, by setting

$$
\left[((x+y)+t)^{k}\right]_{\mathbb{H}^{1}}=\sum_{\substack{h, n_{3}=0,1, \ldots \\ h+2 n_{3}=k}} \frac{k !}{h ! n_{3} !}(x+y)^{h} t^{n_{3}}=\sum_{\substack{n_{1}, n_{2}, n_{3}=0,1, \ldots \\ n_{1}+n_{2}+2 n_{3}=k}} \frac{k !}{n_{1} ! n_{2} ! n_{3} !} x^{n_{1}} y^{n_{2}} t^{n_{3}},
$$

where the $h^{\text {th }}$-power must be computed as for the standard binomial theorem.

These last considerations suggest the following alternative expression for $P_{m, f}$ :

$$
\begin{aligned}
P_{m, f}(x, y, t) & =\sum_{k=0}^{m} \frac{1}{k !}\left[\left(\left(x \frac{\partial}{\partial X}+y \frac{\partial}{\partial X}\right)+t T\right)^{k} f\right]_{\mathbb{H}^{1}}(0)= \\
& =\sum_{k=0}^{m} \frac{1}{k !}\left[\sum_{\substack{h, n_{3}=0,1, \ldots \\
h+2 n_{3}=k}} \frac{k !}{h ! n_{3} !}\left(\left(x \frac{\partial}{\partial X}+y \frac{\partial}{\partial Y}\right)^{h}(t T)^{n_{3}}\right) f\right](0)= \\
\left(P_{m, f} \text { - Third Form }\right) & =\sum_{k=0}^{m}\left[\sum_{\substack{n_{1}, n_{2}, n_{3}=0,1, \ldots \\
n_{1}+n_{2}+2 n_{3}=k}} \frac{\left(\frac{\partial^{n_{1}+n_{2}}}{\partial X^{n_{1}} \partial Y^{n_{2}}} T^{n_{3}} f\right)(0)}{n_{1} ! n_{2} ! n_{3} !} x^{n_{1}} y^{n_{2}} t^{n_{3}}\right] .
\end{aligned}
$$

Setting now,

$$
\mathfrak{S}_{k}:=\left\{\frac{\partial^{n_{1}+n_{2}}}{\partial X^{n_{1}} \partial Y^{n_{2}}} T^{n_{3}}: n_{1}, n_{2}, n_{3}=0,1, \ldots, n_{1}+n_{2}+2 n_{3}=k\right\}
$$


we are going to prove that (see Proposition 1 and Corollary 1 ) the set $\bigcup_{k=0}^{\infty} \mathfrak{S}_{k}$ forms a basis for the vector space of all left invariant differential operators on $\mathbb{H}^{1}$ and that the linear mapping $D: \mathcal{P}_{m} \rightarrow \mathcal{D}_{m}$ defined through the position

$D\left(P_{m}\right)=\sum_{k=0}^{m} \sum_{\substack{n_{1}, n_{2}, n_{3}=0,1, \ldots \\ n_{1}+n_{2}+2 n_{3}=k}}\left(\frac{\partial^{n_{1}+n_{2}}}{\partial X^{n_{1}} \partial Y^{n_{2}}} T^{n_{3}} P_{m}(0)\right) \frac{\partial^{n_{1}+n_{2}}}{\partial X^{n_{1}} \partial Y^{n_{2}}} T^{n_{3}}, \forall P_{m} \in \mathcal{P}_{m}$,

is indeed an isomorphism having a diagonal matrix associated with respect to the two basis

$$
\left\{x^{n_{1}} y^{n_{2}} t^{n_{3}}\right\} \begin{gathered}
n_{1}, n_{2}, n_{3}=0,1, \ldots, m \\
n_{1}+n_{2}+2 n_{3}=0,1, \ldots, m
\end{gathered} \text { and } \bigcup_{k=0}^{m} \mathfrak{S}_{k} .
$$

Roughly speaking, the two bases are essentially dual to each other, i.e., any such a symmetrized derivation acts pointwisely on monomials as if it were an Euclidean iterated derivative. We stress that this result is not at all trivial if one takes into account the very definition of symmetrized derivation as in Definition 9.

We need first the following Lemma 1, whose statement is easily expected if one takes into account the very definition of the vector fields $X$ and $Y$.

Lemma 1 For any $n_{1}, n_{2}, m_{1}, m_{2}=0,1, \ldots$, we have

$$
\left[\frac{\partial^{n_{1}+n_{2}}}{\partial X^{n_{1}} \partial Y^{n_{2}}}\left(x^{m_{1}} y^{m_{2}}\right)\right]_{(x, y, t)=(0,0,0)}=\left\{\begin{array}{cl}
m_{1} ! m_{2} ! & \text { if } n_{1}=m_{1} \text { and } n_{2}=m_{2} \\
0 & \text { otherwise }
\end{array} .\right.
$$

Proof. The proof can be easily achieved by induction on the sum $n_{1}+n_{2}$, taking into account Remark 4.

Proposition 1 For any $n_{1}, n_{2}, n_{3}, m_{1}, m_{2}, m_{3}=0,1, \ldots$, we have

$$
\left[\frac{\partial^{n_{1}+n_{2}}}{\partial X^{n_{1}} \partial Y^{n_{2}}} T^{n_{3}}\left(x^{m_{1}} y^{m_{2}} t^{m_{3}}\right)\right]_{(x, y, t)=(0,0,0)}=\left\{\begin{array}{cl}
m_{1} ! m_{2} ! m_{3} ! & \text { if } n_{i}=m_{i}, i=1,2,3 \\
0 & \text { otherwise }
\end{array} .\right.
$$

Proof. We argue by induction on $n_{3}$, assuming first that the thesis holds when $n_{3}=0$. When $m_{3}=0$ the thesis holds trivially; then, for $m_{3} \geq 1$, we have

$$
\frac{\partial^{n_{1}+n_{2}}}{\partial X^{n_{1}} \partial Y^{n_{2}}} T^{n_{3}}\left(x^{m_{1}} y^{m_{2}} t^{m_{3}}\right)=m_{3} \cdot \frac{\partial^{n_{1}+n_{2}}}{\partial X^{n_{1}} \partial Y^{n_{2}}} T^{n_{3}-1}\left(x^{m_{1}} y^{m_{2}} t^{m_{3}-1}\right),
$$

and the thesis follows easily by the induction hypotheses. So it remains to prove the induction base, i.e. the case $n_{3}=0$. We argue again by induction 
on $n_{1}+n_{2}$. The base $n_{1}+n_{2}=0$ of the induction argument holds trivially, so, according to Remark 4 , assume $n_{1}+n_{2} \geq 1$. When $m_{3}=0$ the thesis easily follows by the previous Lemma 1 ; when $m_{3} \geq 1$ we can write,

$$
\begin{aligned}
{\left[\frac{\partial^{n_{1}+n_{2}}}{\partial X^{n_{1}} \partial Y^{n_{2}}}\left(x^{m_{1}} y^{m_{2}} t^{m_{3}}\right)\right]_{(x, y, t)=(0,0,0)} } & \frac{n_{1}}{n_{1}+n_{2}} \cdot\left[\frac{\partial^{n_{1}+n_{2}-1}}{\partial X^{n_{1}-1} \partial Y^{n_{2}}}\left(X\left(x^{m_{1}} y^{m_{2}}\right) \cdot t^{m_{3}}\right)\right]_{(x, y, t)=(0,0,0)} \\
& +\frac{n_{1}}{n_{1}+n_{2}} \cdot\left[\frac{\partial^{n_{1}+n_{2}-1}}{\partial X^{n_{1}-1} \partial Y^{n_{2}}}\left(x^{m_{1}} y^{m_{2}} \cdot X\left(t^{m_{3}}\right)\right)\right]_{(x, y, t)=(0,0,0)} \\
& +\frac{n_{2}}{n_{1}+n_{2}} \cdot\left[\frac{\partial^{n_{1}+n_{2}-1}}{\partial X^{n_{1}} \partial Y^{n_{2}-1}}\left(Y\left(x^{m_{1}} y^{m_{2}}\right) \cdot t^{m_{3}}\right)\right]_{(x, y, t)=(0,0,0)} \\
& +\frac{n_{2}}{n_{1}+n_{2}} \cdot\left[\frac{\partial^{n_{1}+n_{2}-1}}{\partial X^{n_{1}} \partial Y^{n_{2}-1}}\left(x^{m_{1}} y^{m_{2}} \cdot Y\left(t^{m_{3}}\right)\right)\right]_{(x, y, t)=(0,0,0)} \\
= & \frac{n_{1}}{n_{1}+n_{2}} \cdot\left[\frac{\partial^{n_{1}+n_{2}-1}}{\partial X^{n_{1}-1} \partial Y^{n_{2}}}\left(x^{m_{1}} y^{m_{2}} \cdot X\left(t^{m_{3}}\right)\right)\right]_{(x, y, t)=(0,0,0)} \\
& +\frac{n_{2}}{n_{1}+n_{2}} \cdot\left[\frac{\partial^{n_{1}+n_{2}-1}}{\partial X^{n_{1}} \partial Y^{n_{2}-1}}\left(x^{m_{1}} y^{m_{2}} \cdot Y\left(t^{m_{3}}\right)\right)\right]_{(x, y, t)=(0,0,0)},
\end{aligned}
$$

because by the induction hypotheses (I) and (III) are equal to zero. Now observe that when $m_{3} \geq 2$ also (II) and (IV) are equal to zero. Finally, when $m_{3}=1$, we have

$$
\begin{aligned}
(\mathrm{II})+(\mathrm{IV})= & {\left[\frac{n_{1}}{n_{1}+n_{2}} \cdot \frac{\partial^{n_{1}+n_{2}-1}}{\partial X^{n_{1}-1} \partial Y^{n_{2}}}\left(2 x^{m_{1}} y^{m_{2}+1}\right)\right]_{(x, y, t)=(0,0,0)} } \\
& +\left[\frac{n_{2}}{n_{1}+n_{2}} \cdot \frac{\partial^{n_{1}+n_{2}-1}}{\partial X^{n_{1}} \partial Y^{n_{2}-1}}\left(-2 x^{m_{1}+1} y^{m_{2}}\right)\right]_{(x, y, t)=(0,0,0)} .
\end{aligned}
$$

So, if $n_{1}-1=m_{1}$ and $n_{2}=m_{2}+1$ (otherwise the thesis follows immediately as before), we have

$$
(\mathrm{II})+(\mathrm{IV})=\frac{2\left(m_{1}+1\right)}{m_{1}+m_{2}+2} \cdot m_{1} !\left(m_{2}+1\right) !-\frac{2\left(m_{2}+1\right)}{m_{1}+m_{2}+2} \cdot\left(m_{1}+1\right) ! m_{2} !=0,
$$

that completes the proof.

Corollary 1 The set $\bigcup_{k=0}^{m} \mathfrak{S}_{k}$ is a basis of the vector space of all left invariant differential operators on $\mathbb{H}^{1}$.

Proof. The thesis follows immediately from Proposition 1 and observing that we have,

$$
\left|\mathfrak{S}_{k}\right|=\left|\left\{X^{n_{1}} Y^{n_{2}} T^{n_{3}}\right\}_{\substack{n_{1}, n_{2}, n_{3}=0,1, \ldots \\ n_{1}+n_{2}+2 n_{3}=k}}\right| .
$$


Now we can prove the main result of this section.

Theorem 2 Let $m$ be a non negative integer and let $f \in C_{\mathrm{H}}^{m}\left(\mathbb{H}^{1}\right)$. Then $P_{m, f}$ is the Taylor polynomial of $f$ at zero.

Proof. It suffices to show that $P_{m, f}$ verifies condition ii) of Theorem 1: thanks to Proposition 1 it suffices to check condition ii) with any fixed symmetrized derivation. Having in mind last member of $P_{m, f}$ - Third Form, and thanks to the previous Proposition 1 , for any $n_{1}, n_{2}, n_{3}$ such that $n_{1}+n_{2}+$ $2 n_{3} \leq m$, we have

$$
\begin{aligned}
& {\left[\frac{\partial^{n_{1}+n_{2}}}{\partial X^{n_{1}} \partial Y^{n_{2}}} T^{n_{3}}\left(P_{m, f}-f\right)\right]_{(x, y, t)=(0,0,0)}} \\
& \quad=\left[\frac{\partial^{n_{1}+n_{2}}}{\partial X^{n_{1}} \partial Y^{n_{2}}} T^{n_{3}}\left(\frac{\frac{\partial^{n_{1}+n_{2}}}{\partial X^{n_{1}} \partial Y^{n_{2}}} T^{n_{3}} f(0)}{n_{1} ! n_{2} ! n_{3} !} x^{n_{1}} y^{n_{2}} t^{n_{3}}-f\right)\right]_{(x, y, t)=(0,0,0)} \\
& =\left[\left(\frac{\partial^{n_{1}+n_{2}}}{\partial X^{n_{1}} \partial Y^{n_{2}}} T^{n_{3}} f\right)(0)-\frac{\partial^{n_{1}+n_{2}}}{\partial X^{n_{1}} \partial Y^{n_{2}}} T^{n_{3}} f\right]_{(x, y, t)=(0,0,0)}=0,
\end{aligned}
$$

and the proof is completed.

Before ending this section, we provide an alternative, completely different, proof of Corollary 1 (see Proposition 2) based on some considerations about free Lie algebras. First of all a little bit of notations. Let $\mathbb{K}$ be a field of characteristics zero and let $\mathbb{K}\langle X, Y\rangle$ be the free associative algebra generated by the symbols $X$ and $Y$. It is worth saying that the generators do not commute. The algebra $\mathbb{K}\langle X, Y\rangle$ is naturally graded; indeed, given a monomial $\mathrm{m} \in \mathbb{K}\langle X, Y\rangle$, one can define the degree $\operatorname{deg} \mathrm{m}=$ length $\mathrm{m}$, as the number of the letters of the word which identifies $\mathrm{m}$. For any $k=0,1, \ldots$, we will denote by $\mathbb{K}\langle X, Y\rangle_{k}$ the linear subspace generated by all the monomials $\mathrm{m}$ of degree $k$; so it is $\mathbb{K}\langle X, Y\rangle=\bigoplus_{k=0}^{\infty} \mathbb{K}\langle X, Y\rangle_{k}$, where, clearly, $\operatorname{dim}\left(\mathbb{K}\langle X, Y\rangle_{k}\right)=2^{k}$. A subspace $R$ of $\mathbb{K}\langle X, Y\rangle$ is called homogeneous if, for any polynomial $\mathrm{p}$ in $R$, every homogeneous component of $\mathrm{p}$ is in $R$. It is obvious that, for any $k=0,1, \ldots, \mathbb{K}\langle X, Y\rangle_{k}$ is a homogeneous subspace of $\mathbb{K}\langle X, Y\rangle$ and, moreover, the product mapping $\mathbb{K}\langle X, Y\rangle \times \mathbb{K}\langle X, Y\rangle \ni(\mathrm{p}, \mathrm{q})$ $\rightarrow$ pq $\in \mathbb{K}\langle X, Y\rangle$, maps a product of homogeneous subspaces into another homogeneous subspace; in particular $\mathbb{K}\langle X, Y\rangle_{i} \times \mathbb{K}\langle X, Y\rangle_{j}$ is mapped into $\mathbb{K}\langle X, Y\rangle_{i+j}$. A monomial $\mathrm{m} \in \mathbb{K}\langle X, Y\rangle$ is actually endowed with a partial degree. For any monomial $\mathrm{m} \in \mathbb{K}\langle X, Y\rangle$, define a couple of non-negative integers as follows:

$\operatorname{deg}_{X, Y} \mathrm{~m}=$ ( " number of $X$ in the word $\mathrm{m}$ ", " number of $Y$ in the word $\mathrm{m}$ "). 
For any $i, j=0,1, \ldots$, we will denote with $\mathbb{K}\langle X, Y\rangle_{i, j}$, the subspace of $\mathbb{K}\langle X, Y\rangle_{i+j}$ which is generated by the monomials $\mathrm{m}$ of $\operatorname{deg}_{X, Y} \mathrm{~m}=(i, j)$; a polynomial $\mathrm{p}$ is said to be finely homogeneous if it belongs to $\mathbb{K}\langle X, Y\rangle_{i, j}$. Of course it is $\operatorname{dim}\left(\mathbb{K}\langle X, Y\rangle_{i, j}\right)=\frac{(i+j) !}{i ! j !}$ and, moreover, for any $k=0,1, \ldots$, the subspace $\mathbb{K}\langle X, Y\rangle_{k}$, is decomposed as the direct sum of the subspaces $\mathbb{K}\langle X, Y\rangle_{i, j}$ with $i+j=k$, i.e. $\mathbb{K}\langle X, Y\rangle_{k}=\bigoplus_{i+j=k} \mathbb{K}\langle X, Y\rangle_{i, j}$. Let now $a T$ denote the commutator of $X$ and $Y$, i.e. $a T=[X, Y]=X Y-Y X$, for some $a \neq 0$; the ideal generated by $T$ will be denoted by $(T)$; moreover, as customary, we denote by $\mathbb{K}[x, y]$ the algebra of commutative polynomials. After we define two linear maps $\phi$ and $\psi$ as follows: fix arbitrarily $i, j=$ $0,1, \ldots ;$ The map $\phi: \mathbb{K}\langle X, Y\rangle \rightarrow \mathbb{K}[x, y]$ is the map which associates to each monomial $\mathrm{m} \in \mathbb{K}\langle X, Y\rangle$ of $\operatorname{deg}_{X, Y} \mathrm{~m}=(i, j)$, the monomial $x^{i} y^{j} \in$ $\mathbb{K}[x, y]$. The map $\psi: \mathbb{K}[x, y] \rightarrow \mathbb{K}\langle X, Y\rangle$ is defined on the monomial $x^{i} y^{j}$, as $\psi\left(x^{i} y^{j}\right)=\sigma\left(X^{i}, Y^{j}\right)$ (see Definition 7 ). It is quite easy to prove that ker $\phi=(T)$. Hence we get the following exact sequence of vector spaces

$$
0 \longrightarrow(T) \longrightarrow \mathbb{K}\langle X, Y\rangle \stackrel{\phi}{\longrightarrow} \mathbb{K}[x, y] \longrightarrow 0,
$$

which restricted to homogeneous subspaces gives the following exact sequences of finite dimensional vector spaces

$$
0 \longrightarrow(T)_{k} \longrightarrow \mathbb{K}\langle X, Y\rangle_{k} \stackrel{\phi}{\longrightarrow} \mathbb{K}[x, y]_{k} \longrightarrow 0 .
$$

Since $\phi \circ \psi=\mathrm{Id}$, the sequences of vector spaces split for any $k$ and we get

$$
\mathbb{K}\langle X, Y\rangle_{k}=(T)_{k} \oplus(\operatorname{Im} \psi)_{k} .
$$

From this it follows that the subspace $\mathbb{K}\langle X, Y\rangle_{k}$ will be generated by the $k+1$ canonical generators of $(\operatorname{Im} \psi)_{k}$ and by the polynomials of the form $\mathrm{p} T \mathrm{q}$, where, for any $k=2,3, \ldots, \mathrm{pq}$ is a monomial such that $\operatorname{deg}(\mathrm{pq})=k-2$. It is worth saying that these polynomials do not form a basis. At last, it follows trivially that, if $R \subseteq \mathbb{K}\langle X, Y\rangle$ is an ideal of $\mathbb{K}\langle X, Y\rangle$, we get

$$
\frac{\mathbb{K}\langle X, Y\rangle}{R}=\frac{(T)}{R \cap(T)} \oplus \frac{\operatorname{Im} \psi}{R \cap \operatorname{Im} \psi} .
$$

Furthermore, if $R$ is homogeneous, we get the following equalities:

$$
\frac{\mathbb{K}\langle X, Y\rangle_{k}}{R_{k}}=\frac{(T)_{k}}{R_{k} \cap(T)_{k}} \oplus \frac{(\operatorname{Im} \psi)_{k}}{R_{k} \cap(\operatorname{Im} \psi)_{k}} .
$$

Remark 7 (Direct decomposition in $\left.\mathfrak{h}^{1}\right)$ Let $R=(T X-X T, Y T-$ $T Y)$, then we have, for any $k=0,1, \ldots$,

$$
\frac{\mathbb{K}\langle X, Y\rangle_{k}}{R_{k}}=\frac{(T)_{k}}{R_{k}} \oplus(\operatorname{Im} \psi)_{k} .
$$


Proposition 2 Let $\mathcal{Z}_{0}=\mathfrak{Z}_{0}=\mathfrak{S}_{0}=\{\mathrm{id}\}, \mathcal{Z}_{1}=\mathfrak{Z}_{1}=\mathfrak{S}_{1}=\{X, Y\}$ and according to notations of Section 2 , for $k=2,3, \ldots$,

$$
\begin{aligned}
& \mathcal{Z}_{k}=\left\{Z_{H_{k}}: Z_{H_{k}} \text { is a horizontal } k \text {-derivation }\right\} \\
& \mathfrak{Z}_{k}=\left\{Z_{H_{k}} T, \frac{\partial^{n_{1}+n_{2}}}{\partial X^{n_{1}} \partial Y^{n_{2}}}: Z_{H_{k}} \text { is a horizontal }(k-2)\right. \text {-derivation, } \\
& \left.\qquad n_{1}, n_{2}=0,1, \ldots, n_{1}+n_{2}=k\right\} \\
& \mathfrak{S}_{k}=\left\{\frac{\partial^{n_{1}+n_{2}}}{\partial X^{n_{1}} \partial Y^{n_{2}}} T^{n_{3}}: n_{1}, n_{2}, n_{3}=0,1, \ldots, n_{1}+n_{2}+2 n_{3}=k\right\} .
\end{aligned}
$$

Then, for any $k$ we have

$$
\operatorname{Span}_{\mathbb{R}}\left(\mathcal{Z}_{k}\right)=\operatorname{Span}_{\mathbb{R}}\left(\mathfrak{Z}_{k}\right)=\operatorname{Span}_{\mathbb{R}}\left(\mathfrak{S}_{k}\right) .
$$

Proof. The first equality follows immediately from Remark 7. The second equality follows considering that, for each $k \geq 1$, the elements of $\mathfrak{S}_{k}$ are proved to be linearly independent. Indeed, observe that if

$$
\sum_{n_{1}+n_{2}+2 n_{3}=k} a_{n_{1}, n_{3}, n_{3}} \frac{\partial^{n_{1}+n_{2}}}{\partial X^{n_{1}} \partial Y^{n_{2}}} T^{n_{3}}=0
$$

applying $\phi$ to both members, we recover $\sum_{n_{1}+n_{2}=k} a_{n_{1}, n_{2}, 0} x^{n_{1}} y^{n_{2}}=0$ which implies that any $a_{n_{1}, n_{2}, 0}$ is zero; then (3.3) becomes

$$
\left(\sum_{n_{1}+n_{2}+2 n_{3}=k-2} a_{n_{1}, n_{3}, n_{3}} \frac{\partial^{n_{1}+n_{2}}}{\partial X^{n_{1}} \partial Y^{n_{2}}} T^{n_{3}}\right) T=0
$$

which, in its turn, implies $\sum_{n_{1}+n_{2}+2 n_{3}=k-2} a_{n_{1}, n_{3}, n_{3}} \frac{\partial^{n_{1}+n_{2}}}{\partial X^{n_{1}} \partial Y^{n_{2}}} T^{n_{3}}=0$, and the proof easily follows by induction on $k$.

Remark 8 Observe that another proof of Theorem 2 follows from the standard equality among the derivative of the polynomial of the function and the polynomial of the derivative of the function, working in this case only for the commuting left invariant vector fields: indeed it is easy to verify that for a given $f \in C_{\mathrm{H}}^{m}\left(\mathbb{H}^{1}\right)$, relatively to the polynomial $P_{m, f}$, for any $k=0,1, \ldots,\left[\frac{m}{2}\right]$ we have $T^{k}\left(P_{m, f}-f\right)=P_{m-2 k, T^{k} f}-T^{k} f$, taking into account that $T$ belongs to the center of $\mathfrak{h}^{1}$. Then, through a partial application of Proposition 1, the proof is easily achieved by induction on the degree of the set of generators $\mathfrak{Z}_{k}$.

Remark 9 With very minimal changes all previous considerations work in $\mathbb{H}^{n}$. 


\section{Taylor Formula on step two Carnot groups}

In this section, according with notation introduced in Section 2, we shall denote by $\mathbb{G}$ a step two Carnot group, by $\left\{X_{1}, \ldots, X_{l}\right\}$ a basis of $V_{1}$ and by $\left\{T_{1}, \ldots, T_{p}\right\}$ a basis of $V_{2}$. The arguments employed for the Heisenberg group $\mathbb{H}^{1}$ work as well, with slight changes of notations, in the general case $\operatorname{dim}_{\mathbb{R}}\left(V_{2}\right) \geq 1$. Indeed, arguing as in Section 3, we can recover the Taylor polynomial starting from the Taylor expansion related to a family of $l+p$ left invariant vector fields; more precisely, observing that $V_{2}$ is contained in the center of $\mathfrak{g}$, we define the symmetrized derivation with respect to the vector fields $X_{1}, \ldots, X_{l}, T_{1}, \ldots, T_{p}$ according to the following definition.

Definition 10 Set, for any non negative integers $k, n_{1}, \ldots, n_{l}, s_{1}, \ldots, s_{p}$, such that, $k=n_{1}+\ldots+n_{l}+2\left(s_{1}+\ldots+s_{p}\right)$,

$$
\begin{aligned}
& \frac{\partial^{n_{1}+\cdots+n_{l}}}{\partial X_{1}^{n_{1}} \cdots \partial X_{l}^{n_{l}}} T_{1}^{s_{1}} \cdots T_{p}^{s_{p}}:= \\
& \quad:=\frac{n_{1} ! \cdots n_{l} !}{\left(n_{1}+\cdots+n_{l}\right) !} \cdot \operatorname{Sym}\left(X_{1}^{n_{1}}, \ldots, X_{l}^{n_{l}}\right) T_{1}^{s_{1}} \cdots T_{p}^{s_{p}} .
\end{aligned}
$$

We call (4.1) a symmetrized $k$-derivation of order $k$ containing $n_{1}$-times $X_{1}, \ldots, n_{l}$-times $X_{l}, s_{1}$-times $T_{1}, \ldots, s_{p}$-times $T_{p}$ (symmetrized derivation for short, when we need to specify neither the order $k$ nor the single vector fields).

Remark 10 Formulas (S') and (S") of Section 3 become respectively,

$$
\begin{aligned}
& \operatorname{Sym}\left(X_{1}^{n_{1}}, \ldots, X_{l}^{n_{l}}\right)= \\
& \quad=\operatorname{Sym}\left(X_{1}^{n_{1}-1}, \ldots, X_{l}^{n_{l}}\right) X_{1}+\cdots+\operatorname{Sym}\left(X_{1}^{n_{1}}, \ldots, X_{l}^{n_{l}-1}\right) X_{l},
\end{aligned}
$$

and

$$
\begin{aligned}
\frac{\partial^{n_{1}+\cdots+n_{l}}}{\partial X^{n_{1}} \cdots \partial X^{n_{l}}} & =\frac{n_{1}}{n_{1}+\cdots+n_{l}} \cdot \frac{\partial^{n_{1}+\cdots+n_{l}-1}}{\partial X^{n_{1}-1} \cdots \partial X^{n_{l}}} X_{1}+ \\
& +\cdots \cdots \cdots+\cdots+\cdots+ \\
& +\frac{n_{l}}{n_{1}+\cdots+n_{l}} \cdot \frac{\partial^{n_{1}+\cdots+n_{l}-1}}{\partial X^{n_{1}} \cdots \partial X^{n_{l}-1}} X_{l}
\end{aligned}
$$

as before, the first one is justified by the multinomial identity

$$
\frac{\left(n_{1}+\cdots+n_{l}\right) !}{n_{1} ! \cdots n_{l} !}=\frac{\left(n_{1}+\cdots+n_{l}-1\right) !}{\left(n_{1}-1\right) ! \cdots n_{l} !}+\cdots+\frac{\left(n_{1}+\cdots+n_{l}-1\right) !}{n_{1} ! \cdots\left(n_{l}-1\right) !},
$$

while the second one follows immediately by the first one after some easy calculations. 
In this case, the right formula for the Taylor polynomial for a given $f \in C_{\mathrm{H}}^{m}(\mathbb{G})$, comes to be

$\left(P_{m, f}\right.$ - Step-2 Groups $) \quad P_{m, f}(z)=\sum_{k=0}^{m}\left[\sum_{d(I)=k} \frac{\left(\frac{\partial^{n_{1}+\cdots+n_{l}}}{\left.\partial X_{1}^{n_{1} \cdots \partial X_{l}^{n_{l}}} T_{1}^{m_{1}} \cdots T_{p}^{m_{p}} f\right)(0)}\right.}{n_{1} ! \cdots n_{l} ! m_{1} ! \cdots m_{p} !} z^{I}\right]$,

where, for any $k=0, \ldots, m, I=\left(n_{1}, \ldots, n_{l}, m_{1}, \ldots, m_{p}\right)$ denotes a general $(l+p)$-tuple of non negative integers, $d(I)=n_{1}+\ldots+n_{l}+2\left(m_{1}+\ldots+m_{p}\right)$ and $z^{I}=x_{1}^{n_{1}} \cdots x_{l}^{n_{l}} t_{1}^{m_{1}} \cdots t_{p}^{m_{p}}$.

Now we set,

$$
\begin{aligned}
\mathfrak{S}_{k}= & \left\{\frac{\partial^{n_{1}+\cdots+n_{l}}}{\partial X_{1}^{n_{1}} \cdots \partial X_{l}^{n_{l}}} T_{1}^{s_{1}} \cdots T_{p}^{s_{p}}:\right. \\
& \left.n_{1}, \ldots, n_{l}, s_{1}, \ldots, s_{p}=0,1, \ldots, n_{1}+\cdots+n_{l}+2\left(s_{1}+\cdots+s_{p}\right)=k\right\}
\end{aligned}
$$

with $\mathfrak{S}_{0}=\{$ id $\}$.

We check that the set $\bigcup_{k=0}^{m} \mathfrak{S}_{k}$ is a basis of the vector space of all differential operators on $\mathbb{G}$ and that the Taylor polynomial $P_{m, f}$ - Step-2 Groups satisfy $\left.i i\right)$ of Theorem 1. To this aim, it suffices to extend Lemma 1 and Proposition 1.

Lemma 2 For any $n_{1}, \ldots, n_{l}, m_{1}, \ldots, m_{l}=0,1, \ldots$, we have,

$$
\begin{gathered}
{\left[\frac{\partial^{n_{1}+\ldots+n_{l}}}{\partial X_{1}^{n_{1}} \cdots \partial X_{l}^{n_{l}}} x_{1}^{m_{1}} \cdots x_{l}^{m_{l}}\right]_{\left(x_{1}, \ldots, x_{l}\right)=(0, \ldots, 0)}} \\
\quad=\left\{\begin{array}{cl}
m_{1} ! \cdots m_{l} ! & \text { if } n_{1}=m_{1} \ldots n_{l}=m_{l} \\
0 & \text { otherwise }
\end{array} .\right.
\end{gathered}
$$

Proof. The proof can be easily achieved by induction on the sum $n_{1}+\cdots+n_{l}$, taking into account $\left(\mathrm{S}_{2}\right)$ in Remark 10.

Proposition 3 For any $n_{1}, \ldots, n_{l}, s_{1}, \ldots, s_{p}, m_{1}, \ldots, m_{l}, r_{1}, \ldots, r_{p}$, we have

$$
\begin{aligned}
& {\left[\frac{\partial^{n_{1}+\cdots+n_{l}}}{\partial X^{n_{1}} \cdots \partial X^{n_{l}}} T_{1}^{s_{1}} \cdots T_{p}^{s_{p}}\left(x_{1}^{m_{1}} \cdots x_{l}^{m_{l}} t_{1}^{r_{1}} \cdots t_{p}^{r_{p}}\right)\right]_{\left(x_{1}, \ldots, x_{l}, t_{1}, \ldots, t_{p}\right)=(0, \ldots, 0)}} \\
& =\left\{\begin{array}{cl}
m_{1} ! \cdots m_{l} ! r_{1} ! \cdots r_{p} ! & \text { if } n_{i}=m_{i}, i=1, \ldots, l \text { and } s_{i}=r_{i}, i=1, \ldots, p \\
0 & \text { otherwise. }
\end{array}\right.
\end{aligned}
$$

Proof. We argue by induction on $s_{1}+\cdots+s_{p}$, assuming first that the thesis holds when $s_{1}+\cdots+s_{p}=0$. Then, suppose $s_{1}+\cdots+s_{p} \geq 1$. If $r_{1}+\cdots+r_{p}=0$, the thesis holds trivially. If $r_{1}+\cdots+r_{p} \geq 1$, we can 
suppose, without loss of generality, that $s_{p} \geq 1$; then, supposing $r_{p} \geq 1$, we have,

$$
\begin{aligned}
T_{1}^{s_{1}} \cdots T_{p}^{s_{p}}\left(x_{1}^{m_{1}} \cdots\right. & \left.x_{l}^{m_{l}} t_{1}^{r_{1}} \cdots t_{p}^{r_{p}}\right)= \\
& = \begin{cases}r_{p} \cdot T_{1}^{s_{1}} \cdots T_{p}^{s_{p}-1}\left(x^{m_{1}} \cdots x^{m_{l}} t_{1}^{r_{1}} \cdots t_{p}^{r_{p}-1}\right) & \text { if } r_{p}=s_{p} \\
0 & \text { if } r_{p} \neq s_{p}\end{cases}
\end{aligned}
$$

and the thesis follows by the induction hypothesis. So, it remains to prove the induction base, i.e., the case $s_{1}+\cdots+s_{p}=0$. We argue, again, by induction on $n_{1}+\cdots+n_{l}$. The basis $n_{1}+\cdots+n_{l}=0$ of the induction argument holds trivially, so, assume $n_{1}+\cdots+n_{l} \geq 1$. Then, if $r_{1}+\cdots+r_{p}=0$, the thesis follows by Lemma 2 ; if $r_{1}+\cdots+r_{p} \geq 1$, observe that, according to $\left(\mathrm{S}_{2}\right)$ of Remark 10, we can write, for each $j=1, \ldots, p$,

$$
\begin{aligned}
\frac{\partial^{n_{1}+\cdots+n_{l}}}{\partial X_{1}^{n_{1}} \cdots \partial X_{l}^{n_{l}}}( & \left.x_{1}^{m_{1}} \cdots x_{l}^{m_{l}} t_{j}\right)= \\
= & \frac{n_{1}}{n_{1}+\cdots+n_{l}} \cdot \frac{\partial^{n_{1}+\cdots+n_{p}-1}}{\partial X_{1}^{n_{1}-1} \cdots \partial X_{l}^{n_{l}}} X_{1}\left(x_{1}^{m_{1}} \cdots x_{l}^{m_{l}} t_{j}\right)+ \\
& +\cdots+\frac{n_{l}}{n_{1}+\cdots+n_{l}} \cdot \frac{\partial^{n_{1}+\cdots+n_{l}-1}}{\partial X_{1}^{n_{1}} \cdots \partial X_{l}^{n_{l}-1}} X_{l}\left(x_{1}^{m_{1}} \cdots x_{l}^{m_{l}} t_{j}\right) .
\end{aligned}
$$

Now, to achieve the thesis, it is sufficient to show that, for any $n_{1}, \ldots, n_{l}$, $m_{1}, \ldots, m_{l}=0,1, \ldots$, for each $j=1, \ldots, p$, we have:

$$
\left[\frac{\partial^{n_{1}+\cdots+n_{l}}}{\partial X_{1}^{n_{1}} \cdots \partial X_{l}^{n_{l}}}\left(x_{1}^{m_{1}} \cdots x_{l}^{m_{l}} t_{j}\right)\right]_{\left(x_{1}, \ldots, x_{l}, t_{1}, \ldots, t_{p}\right)=(0, \ldots, 0)}=0 .
$$

Indeed, if we apply, for each $i=1, \ldots, l$, the vector field $X_{i}$ to $x_{1}^{m_{1}} \cdots x_{l}^{m_{l}} t_{j}$, we get $X_{i}\left(x_{1}^{m_{1}} \cdots x_{l}^{m_{l}} t_{j}\right)=X_{i}\left(x_{1}^{m_{1}} \cdots x_{l}^{m_{l}}\right) t_{j}+x_{1}^{m_{1}} \cdots x_{l}^{m_{l}} X_{i}\left(t_{j}\right):=(\mathrm{I})_{i}+(\mathrm{II})_{i}$ and, by the induction hypothesis, it follows that any symmetrized derivation in the right hand side of (4.2) applied to the respective $(\mathrm{I})_{i}$ is zero when evaluated at the origin. So, we have

$$
\begin{aligned}
& {\left[\frac{\partial^{n_{1}+\cdots+n_{l}}}{\partial X_{1}^{n_{1}} \cdots \partial X_{l}^{n_{l}}}\left(x_{1}^{m_{1}} \cdots x_{l}^{m_{l}} t_{j}\right)\right]_{\left(x_{1}, \ldots, x_{l}, t_{1}, \ldots, t_{p}\right)=(0, \ldots, 0)}=} \\
& =\frac{n_{1}}{n_{1}+\cdots+n_{l}} \cdot\left[\frac{\partial^{n_{1}+\cdots+n_{l}-1}}{\partial X_{1}^{n_{1}-1} \cdots \partial X_{l}^{n_{l}}}\left(x_{1}^{m_{1}} \cdots x_{l}^{m_{l}} X_{1}\left(t_{j}\right)\right)\right]_{\left(x_{1}, \ldots, x_{l}, t_{1}, \ldots, t_{p}\right)=(0, \ldots, 0)} \\
& +\cdots \cdots \cdots \cdots \cdots \cdots \cdots+
\end{aligned}
$$

$$
+\frac{n_{l}}{n_{1}+\cdots+n_{l}} \cdot\left[\frac{\partial^{n_{1}+\cdots+n_{l}-1}}{\partial X_{1}^{n_{1}} \cdots \partial X_{l}^{n_{l}-1}}\left(x_{1}^{m_{1}} \cdots x_{l}^{m_{l}} X_{l}\left(t_{j}\right)\right)\right]_{\left(x_{1}, \ldots, x_{l}, t_{1}, \ldots, t_{p}\right)=(0, \ldots, 0)} .
$$


Now, taking into account the form of the vector field $X_{h}$ we have, for each fixed $h=1, \ldots, l$,

$$
X_{h}\left(t_{j}\right)=\frac{1}{2}\left(b_{h 1}^{j} x_{1}+\cdots+b_{h l}^{j} x_{l}\right) .
$$

Then, by Lemma 2, there exists at most one $k \in\{1, \ldots, l\}$ (suppose $h \leq k$ ), such that

$$
\left(n_{1}, \ldots, n_{h}-1, \ldots, n_{k}, \ldots, n_{l}\right)=\left(m_{1}, \ldots, m_{h}, \ldots, m_{k}+1, \ldots, m_{l}\right)
$$

and, in turn, such that

$$
\begin{aligned}
& \quad \frac{n_{h}}{n_{1}+\cdots+n_{l}} \cdot \frac{\partial^{n_{1}+\cdots+n_{l}-1}}{\partial X^{n_{1}} \cdots \partial X^{n_{h}-1} \cdots \partial X^{n_{l}}}\left(x_{1}^{m_{1}} \cdots x_{l}^{m_{l}} X_{h}\left(t_{j}\right)\right)= \\
& =\frac{n_{h}}{n_{1}+\cdots+n_{l}} \cdot \\
& \quad \cdot \frac{\partial^{n_{1}+\cdots+n_{l}-1}}{\partial X^{n_{1}} \cdots \partial X^{n_{h}-1} \cdots \partial X^{n_{l}}}\left(\frac{1}{2} \cdot b_{h k}^{j} \cdot\left(x_{1}^{m_{1}} \cdots x_{h}^{m_{h}} \cdots x_{k}^{m_{k}+1} \cdots x_{l}^{m_{l}}\right)\right) \\
& =\frac{1}{2} \cdot b_{h k}^{j} \cdot \frac{m_{1} ! \cdots\left(m_{h}+1\right) ! \cdots\left(m_{k}+1\right) ! \ldots, m_{l} !}{m_{1}+\cdots+m_{l}+2} .
\end{aligned}
$$

To conclude the proof, we notice that (4.5) is equivalent to

$$
\left(n_{1}, \ldots, n_{h}, \ldots, n_{k}-1, \ldots, n_{l}\right)=\left(m_{1}, \ldots, m_{h}+1, \ldots, m_{k}, \ldots, m_{l}\right)
$$

so, it suffices to observe that, by similar calculations on the term

$$
\frac{n_{h}}{n_{1}+\cdots+n_{l}} \cdot \frac{\partial^{n_{1}+\cdots+n_{l}-1}}{\partial X^{n_{1}} \cdots \partial X^{n_{k}-1} \cdots \partial X^{n_{l}-1}}\left(x_{1}^{m_{1}} \cdots x_{l}^{m_{l}} X_{k}\left(t_{j}\right)\right),
$$

we obtain a pair of terms that cancel out since the matrices $B^{j}$ are skewsymmetric. Then, summing all terms in (4.3), we obtain zero, as desired.

\section{References}

[1] Arena, G. And Caruso, A. O.: Higher order calculus on step two Carnot groups and applications. Preprint, 2008.

[2] Bellaïche, A. And Risler, J-J.: Sub-Riemannian geometry. Progress in Mathematics 144. Birkhäuser Verlag, Basel, 1996.

[3] Bieske, T.: On $\infty$-harmonic functions on the Heisenberg group. Comm. Partial Differential Equations 27 (2002), no. 3-4, 727-761.

[4] Bonfiglioli, A., Lanconelli, E. And Uguzzoni, F.: Stratified Lie groups and potential theory for their sub-Laplacians. Springer Monographs in Mathematics. Springer, Berlin, 2007.

[5] Federer, H.: Geometric measure theory. Classics in Mathematics. Springer, 1996. 
[6] Folland, G. B. And Stein, E. M.: Hardy spaces on homogeneous groups. Mathematical Notes 28. Princeton University Press and University of Tokyo Press, 1982.

[7] Franchi, B., Serapioni, R. and Serra Cassano, F.: Rectifiability and perimeter in the Heisenberg Group. Math. Ann. 321 (2001), 479-531.

[8] Franchi, B., Serapioni, R. and Serra Cassano, F.: On the structure of finite perimeter sets in step 2 Carnot groups. J. Geom. Anal. 13 (2003), no. 3, 421-466.

[9] Kaplan, A.: Fundamental solutions for a class of hypoelliptic PDE generated by composition of quadratic forms. Trans. Amer. Math. Soc. 258 (1980), no. 1, 147-153.

[10] Nagel, A., Stein, E. M. And Wainger, S.: Ball and metrics defined by vector fields. I. Basic properties. Acta Math. 155 (1985), 103-147.

[11] Varadarajan, V.S.: Lie groups, Lie algebras, and their representations. Graduate Texts in Mathematics 102. Springer-Verlag, New York, 1984.

[12] Whitney, H.: Analitic extensions of differentiable functions defined in closed sets. Trans. Amer. Math. Soc. 36 (1934), no. 1, 63-89.

Recibido: 9 de abril de 2008

Gabriella Arena

Dipartimento di Matematica e Informatica

Università di Catania

Viale A. Doria 6, 95125, Catania, Italy http://www.dmi.unict.it/ garena

garena@dmi.unict.it

Andrea O. Caruso

Dipartimento di Matematica e Informatica

Università di Catania

Viale A. Doria 6-I, 95125, Catania, Italy http://www.dmi.unict.it/ aocaruso aocaruso@dmi.unict.it

Antonio Causa

Dipartimento di Matematica e Informatica Università di Catania Viale A. Doria 6-I, 95125, Catania, Italy causa@dmi.unict.it

G. Arena is supported by MIUR (Italy), by I.N.d.A.M. and by University of Trento. A. O. Caruso is supported by MIUR (Italy) and by University of Catania. 